\title{
Obstrução duodenal por hematoma parietal pós-trauma: relato de caso e revisão da literatura*
}

\author{
Post-traumatic duodenal obstruction by intramural hematoma: a case report and literature review
}

Henrique Silva Thé Pontes ${ }^{1}$, Eugênio Albuquerque Pequeno ${ }^{2}$

Resumo Os hematomas parietais de alças intestinais por trauma abdominal fechado, determinando rápida obstrução luminal, são lesões raras e podem ser confundidas com afecções neoplásicas estenosantes (parietais ou por mecanismo de compressão extrínseca). Neste estudo é relatado um caso de obstrução intestinal por hematoma parietal da terceira porção duodenal pós-trauma e são discutidos o diagnóstico por imagem e o tratamento para tal obstrução.

Unitermos: Hematoma; Trauma abdominal fechado; Obstrução intestinal.

Abstract Intramural duodenal hematomas secondary to blunt trauma, determining rapid luminal obstruction, are rare, benign lesions and may be confused with obstructive neoplastic conditions (either parietal or produced by extrinsic compression mechanisms). The present report describes a case of post-traumatic intestinal obstruction by an intramural hematoma in the third duodenal segment. Imaging diagnosis and the conservative management of such obstruction are discussed. Keywords: Hematoma; Blunt abdominal trauma; Intestinal obstruction.

Pontes HST, Pequeno EA. Obstrução duodenal por hematoma parietal pós-trauma: relato de caso e revisão da literatura. Radiol Bras. 2012 Jul/Ago;45(4):235-237.

\section{INTRODUÇÃO}

O hematoma intramural duodenal (HIMD) é causa incomum de obstrução intestinal alta em crianças com história de trauma abdominal fechado. Esses traumas são algumas vezes triviais que, em alguns casos, podem ser esquecidos pela criança ou, outras vezes, são tão violentos a ponto de esses pacientes ocultá-los, por medo de receberem represália por parte dos pais.

O objetivo deste trabalho é que, mesmo não dispondo de informações clínicas precisas e sabendo que não existem achados patognomônicos de imagem para o hematoma parietal, diante de um quadro obstrutivo intestinal alto e agudo no grupo pediátrico nós devemos sempre incluir no diagnóstico diferencial a possibilidade de hematoma mural do duodeno, levando em

* Trabalho realizado no Hospital Infantil Luís França, Fortaleza, $\mathrm{CE}$, Brasil.

1. Médico Radiologista, Chefe do Serviço de Ultrassonografia Petite Image do Hospital Infantil Luís França, Fortaleza, CE, Brasil.

2. Médico Radiologista, Chefe do Serviço de Radiologia do Hospital Infantil Luís França, Fortaleza, CE, Brasil.

Endereço para correspondência: Dr. Henrique Silva Thé Pontes. Hospital Infantil Luís França. Avenida Heráclito Graça, 60, Centro. Fortaleza, CE, Brasil, 60140-060. E-mail: henriquesilvathe@ terra.com.br

Recebido para publicação em 17/8/2011. Aceito, após revisão, em 1/3/2012. conta os critérios de adequação de exames radiológicos no grupo pediátrico.

\section{RELATO DO CASO}

Paciente do sexo feminino, seis anos de idade, branca, natural de Mossoró, RN, atendida no Pronto-Socorro do Hospital Infantil Luís França, no município de Fortaleza, CE, em 30 de junho de 2011, apresentando quadro de obstrução intestinal alta (vômitos biliosos pós-prandiais há dois dias, sinais de desidratação leve e dor na região abdominal superior). Os responsáveis pela criança negavam sintomas semelhantes anteriormente e/ou doença prévia conhecida. Não tinha perda de peso ou história de maus tratos.

Foram realizados, no dia da admissão hospitalar (30/6/2011), hemograma completo e radiografia do tórax, que resultaram normais, e intempestivamente, endoscopia digestiva alta, que alcançou até a primeira porção duodenal, revelando líquido de estase gástrica com discreto processo inflamatório, não fazendo referência às demais porções duodenais.

No dia seguinte (1/7/2011) foi realizada seriografia esofagogastroduodenal, que demonstrou obstrução total na transição da segunda para a terceira porção duodenal com parada total da progressão do meio de contraste, indicando obstrução luminal por espessamento parietal (Figura 1), e em seguida foi realizada ultrassonografia abdominal, que evidenciou lesão hipoecoica, sólida, na região retroperitoneal da metade superior do abdome, ao nível da linha mediana, entre os grandes vasos (veia cava inferior/aorta) e a glândula pancreática normal. A lesão determinava rechaço anterior

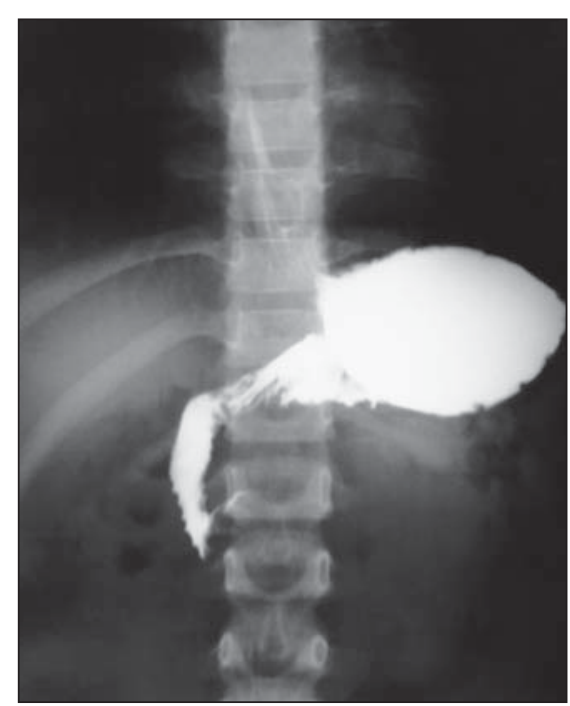

Figura 1. Seriografia esofagogastroduodenal revela obstrução total na transição da segunda para a terceira porção duodenal, com parada total da progressão do contraste. 


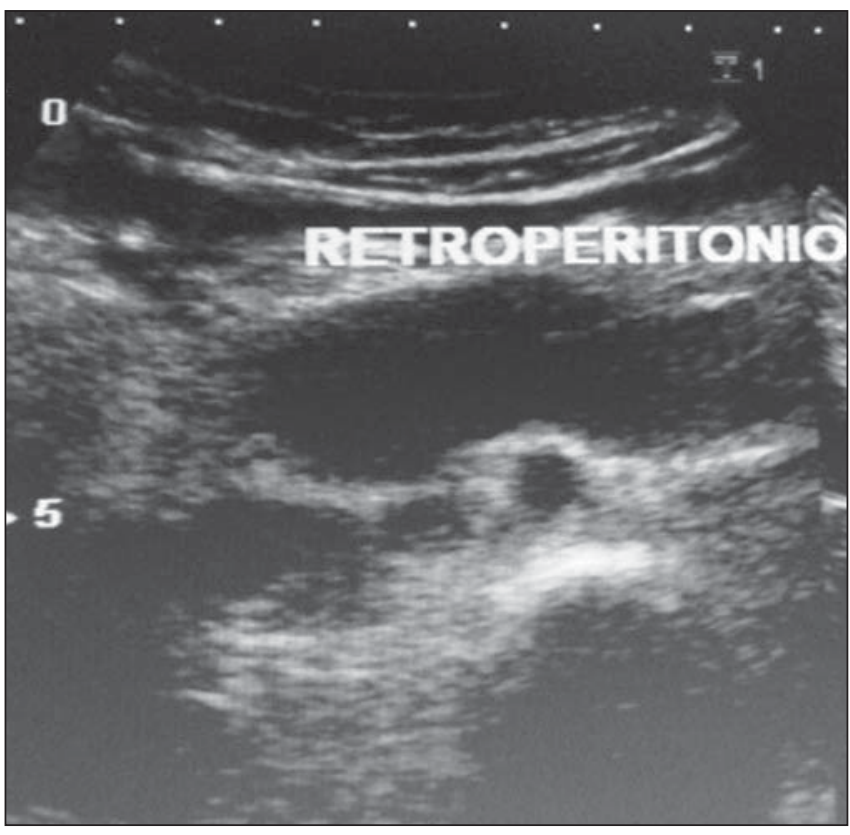

Figura 2. Ultrassonografia abdominal mostra imagem de lesão hipoecoica (5,5 $\times 2,3 \mathrm{~cm}$ ) retroperitoneal na metade superior do abdome, mediana, entre os grandes vasos (veia cava inferior/aorta) e a glândula pancreática normal. A lesão determinava obstrução ao trânsito duodenal (peristaltismo de luta da segunda porção e refluxo de líquido para o estômago), líquido de estase gástrica, que serviu de janela acústica.

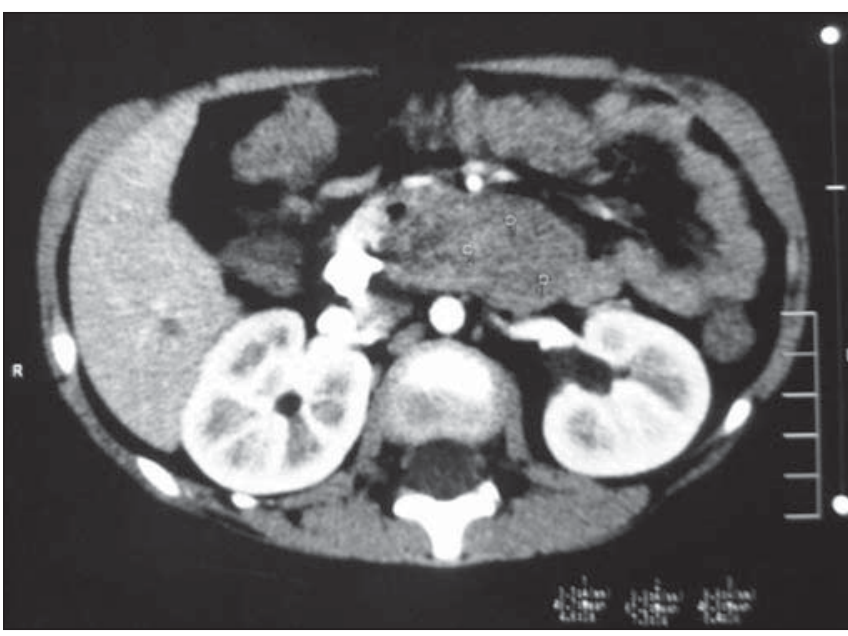

Figura 3. Tomografia multislice pós-contraste evidencia lesão expansiva hipodensa com leve captação do produto de contraste em sítio retroperitoneal (provável massa linfonodal) determinando compressão extrínseca e/ou invasão duodenal por contiguidade. da artéria mesentérica superior e obstrução ao trânsito duodenal (peristaltismo de luta da segunda porção com refluxo de líquido para o estômago, e estase líquida gástrica, que serviu de janela acústica) e media 5,5 $\times 2,3 \mathrm{~cm}$. Não foi observado fluxo sanguíneo intralesional ao estudo Doppler colorido, sendo a lesão sugestiva de hematoma parietal duodenal obstrutivo pós-traumático, apesar de não haver relato de trauma ou hematoma na pele da região epigástrica (Figura 2). Com isso, foi introduzida sonda nasogástrica para descompressão do estômago, hidratação parenteral (venosa) e solicitada tomografia computadorizada para melhor caracterizar o quadro.

No segundo dia de internação hospita$\operatorname{lar}(2 / 7 / 2011)$ foi realizada, em outro serviço, tomografia computadorizada multislice do abdome superior antes e após administração de contraste intravenoso, que evidenciou lesão expansiva hipodensa, a qual apresentava leve captação do produto de contraste, localizada em sítio retroperitoneal, determinando compressão extrínseca e/ou invasão duodenal por continuidade (lesão de natureza neoplásica linfoproliferativa determinando obstrução duodenal) (Figura 3).
Com a hipótese diagnóstica de neoplasia linfoproliferativa determinando oclusão duodenal, foi indicada intervenção cirúrgica para biopsiar a lesão e proporcionar tratamento do quadro obstrutivo. Imediatamente antes da intervenção (5/7/2011) houve informação de que um adulto testemunhara violento trauma abdominal (guidão de bicicleta) sofrido pela criança dois dias antes da internação hospitalar. Em virtude do bom estado geral da paciente e dessa nova informação de trauma, foi suspensa a operação, sendo indicados alimentação parenteral e tratamento expectante.

A paciente teve boa evolução, não mais havendo drenagem de suco gastrobiliar pela sonda, nem dor abdominal. Foi então realizada seriografia gastroduodenal de controle no $9^{\circ}$ dia de internação (8/7/2011), que evidenciou trânsito duodenal livre de obstáculos (Figura 4). A paciente recebeu alta hospitalar, curada, no $10^{\circ}$ dia.

\section{DISCUSSÃO}

O HIMD traumático em crianças ocorre em cerca de $2 \%$ a $3 \%$ dos traumas abdominais fechados ${ }^{(\mathbf{1 , 2})}$. Fatores anatômicos como fixação retroperitoneal do duodeno, posi-

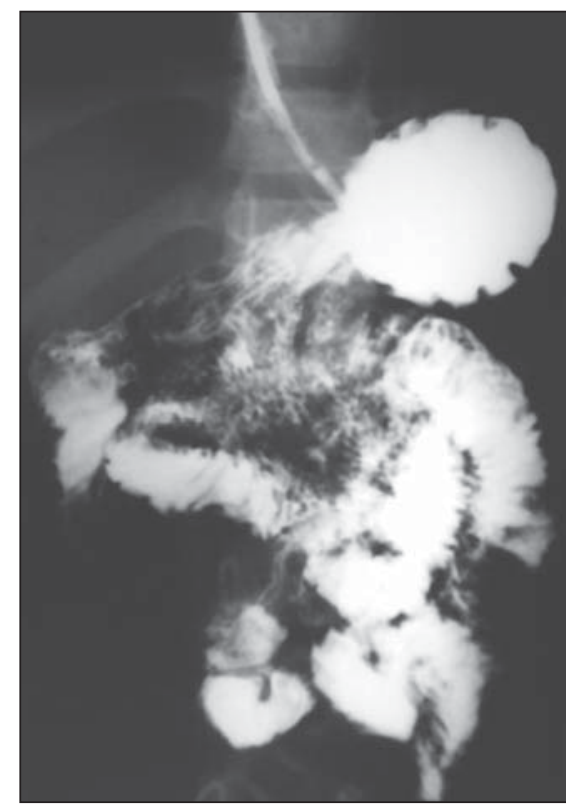

Figura 4. Seriografia gastroduodenal de controle demonstra trânsito duodenal se processando livremente em tempo fisiológico, sem obstáculos.

ção em frente à coluna lombar, um rico plexo vascular subseroso/submucoso e uma fraca musculatura da parede abdominal anterior, todas essas razões contribuem para o aparecimento do hematoma intramural duodenal. 
O hematoma mural do duodeno pode incidir em todas as idades e em ambos os sexos, mas no grupo pediátrico os meninos são mais afetados ${ }^{(3)}$. A relação de proximidade do duodeno com o pâncreas explica por que a pancreatite traumática é a comorbidade mais frequente associada ao hematoma intramural duodenal. Jewett et al. ${ }^{(1)}$ apontaram $21 \%$ de pancreatite associada, em um estudo de revisão de 182 casos de hematoma parietal duodenal em crianças. No entanto, o trauma, algumas vezes, é trivial, podendo algumas crianças esquecer de relatá-lo ${ }^{(4)}$. Traumas causados por guidão de bicicleta, acidente de trânsito e esportes violentos são os principais fatores etiológicos ${ }^{(\mathbf{4 , 5})}$. Maus tratos em crianças sempre devem ser incluídos como fator causal, principalmente em crianças menores de cinco anos ${ }^{(6,7)}$.

Nos portadores de coagulopatias, subgrupo especial, apresenta-se como um fator etiológico em traumas mínimos e procedimentos endoscópicos $^{\left({ }^{(8)}\right.}$. Isso permitiu sugerir a indicação dos procedimentos endoscópicos em crianças somente quando as plaquetas estiverem acima de $50.000 / \mathrm{mm}^{3}$, com tempo de protrombina e tempo parcial de tromboplastina normais ${ }^{(\boldsymbol{9})}$.

A fisiopatologia do HIMD por trauma deve-se ao choque da alça contra a coluna lombar por um agente traumático, que resulta em contusão, sangramento parietal que gera um hematoma. Esse hematoma pode crescer e gradualmente provocar obstrução luminal do órgão.

O diagnóstico de HIMD é baseado nos sinais e sintomas de obstrução intestinal alta, em seguida a um trauma, respaldados por evidências radiológicas de obstrução intestinal $^{(\mathbf{1 0})}$. O método de imagem de es- colha para HIMD é a tomografia computadorizada com contraste; entretanto, a ecografia é um método valioso, não invasivo, que fornece dados importantes sobre o aspecto mural da lesão. O estudo endoscópico e a ressonância magnética podem ser realizados em casos isolados quando os dados clínicos e os exames convencionais são inconclusivos.

No presente caso houve uma certa inversão na sequência dos exames complementares, em razão de o serviço não dispor, no momento, de tomógrafo.

O tratamento conservador consiste em descompressão gástrica (sonda nasogástrica) e alimentação parenteral. Distúrbios da coagulação devem ser excluídos ou tratados. Se a pancreatite está presente, deve ser devidamente tratada. O seguimento clínico pode ser realizado por imagens de ultrassonografia. Após alguns dias, usualmente uma semana, a sonda nasogástrica pode ser periodicamente fechada para teste e estimulação do trânsito duodenal. Líquidos claros são introduzidos e a dieta é gradualmente restabelecida (líquida restrita $\rightarrow$ pastosa $\rightarrow$ branda $\rightarrow$ geral). Na maioria dos casos o hematoma se resolve em torno de duas semanas de tratamento. O tratamento cirúrgico está indicado se não há melhora clínica ou se o hematoma não se resolve em duas semanas. $\mathrm{O}$ tratamento conservador do HIMD é altamente bem sucedido, principalmente quando o diagnóstico é precocemente realizado.

Em relação ao caso aqui exposto, devese levar em conta duas situações que possibilitam reduzir erros diagnósticos. Em primeiro lugar, pensar nas condições de negação do trauma abdominal, e que um trauma brutal, bruscamente deferido com extrema violência/velocidade pode danificar um órgão ou estrutura interna sem provocar danos na pele da parede onde foi aplicado o golpe - como são instruídos os praticantes de lutas marciais. Por isso, a comunidade médica deve, por todos esses aspectos, ficar atenta para incluir essa possibilidade diagnóstica (hematoma mural do duodeno), mesmo que remotamente, diante de um quadro de obstrução intestinal alta.

\section{REFERÊNCIAS}

1. Jewett TC Jr, Caldarola V, Karp MP, et al. Intramural hematoma of the duodenum. Arch Surg. 1988;123:54-8.

2. Kaufman RA, Towbin R, Babcock DS, et al. Abdominal upper trauma in children: imaging evaluation. AJR Am J Roentgenol. 1984;142:449-60.

3. Felson B, Levin EJ. Intramural hematoma of the duodenum: a diagnostic roentgen sign. Radiology. 1954;63:823-31.

4. Bass D. Duodenal hematoma occurs in children with bicycle injuries. BMJ. 2001;323:754-5.

5. Lam JP, Eunson GJ, Munro FD, et al. Delayed presentation of handlebar injuries in children. BMJ. 2001;322:1288-9.

6. Andersson A, Bergdahl L. Intramural hematoma of the duodenum in children: review of literature and report of two cases. Am Surg. 1973;39:4025.

7. Woolley MM, Mahour GH, Sloan T. Duodenal hematoma in infancy and childhood: changing etiology and changing treatment. Am J Surg. 1978; 136:8-14.

8. Karjoo M, Luisiri A, Silberstein M, et al. Duodenal hematoma and acute pancreatitis after upper gastrointestinal endoscopy. Gastrointest Endosc. 1994;40:493-5.

9. Ramakrishna J, Treem WR. Duodenal hematoma as a complication of endoscopic biopsy in pediatric bone marrow transplant recipients. J Pediatr Gastroenterol Nutr. 1997;25:426-9.

10. Maull KI, Fallahzadeh H, Mays ET. Selective management of post-traumatic obstructing intramural hematoma of the duodenum. Surg Gynecol Obstet. 1978;146:221-4. 